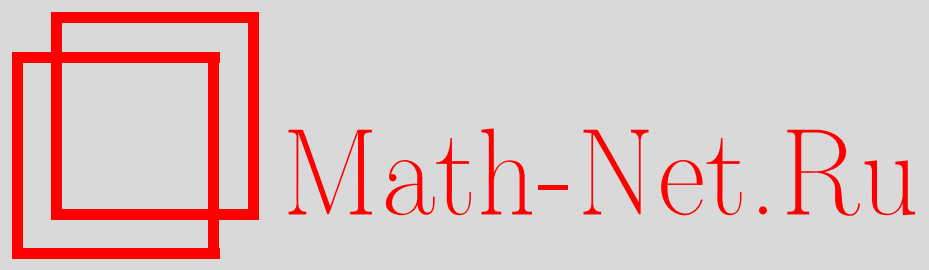

В. Л. Левин, Существование и единственность сохраняющего меру оптимального отображения в общей задаче Монжа-Канторовича, Функи. анализ и его прил., 1998, том 32, выпуск 3, 79-82

DOI: https://doi.org/10.4213/faa431

Использование Общероссийского математического портала MathNet.Ru подразумевает, что вы прочитали и согласны с пользовательским соглашением http://www.mathnet.ru/rus/agreement

Параметры загрузки:

IP : 54.147 .182 .235

26 апреля 2023 г., 16:05:56

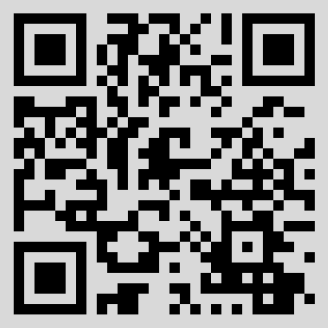


3. Теорема 1 может быть обобщена для интерполяции по симметрическим функциям [6]. Мы займемся этим в другой статье.

4. Условия гладкости на интерполирующий набор функций в теореме 1 могут быть ослаблены, но для ясности мы жертвуем несущественной общностью.

\title{
ЛИТЕРАТУРА
}

1. Арнольд В. И. УМН, 47, вып. 1, 3-45 (1992). 2. Гельфонд А. О. Исчисление конечных разностей. Наука, М., 1967. 3. Ильюта Г. Г. Доклады РАН, 347, № 3, 217-219 (1996). 4. Ильюта Г. Г. Изв. РАН, сер. матем., 60, вып. 6, 91-100 (1996). 5. Bjorner A., Las Vergnas M., Sturmfels B., White N., Ziegler G. Oriented matroids. Cambridge Univ. Press, Cambridge, 1993. 6. Chen W., Louck J. Adv. Math., 117, 147-156 (1996). 7. Kapranov M., Voevodsky V. Combinatorial geometric aspects of polycategory theory: pasting schemes and higher Bruhat orders. Cahiers de Topol. et Geom., 1991, v. XXXII-1, pp. 11-27. 8. Manin Yu., Schechtman V. Adv. Stud. Pure Math., 17, 289-308 (1989). 9. Ziegler G. Topology, 32, No. 2, 259-279 (1993).

Государственная академия

Поступило в редакцию нефти и газа им. И. М. Губкина

2 апреля 1997 г.

УДК 517.9

\section{Существование и единственность сохраняющего меру оптимального отображения в общей задаче Монжа-Канторовича}

\author{
(c) 1998. В. Л. ЛЕвин
}

1. Введение. Пусть $X, Y$ - польские пространства, $\sigma_{1}$ и $\sigma_{2}$ - конечные положительные борелевские меры на них с $\sigma_{1} X=\sigma_{2} Y$ и $c$ - непрерывная функция на $X \times Y$. Далее $\pi_{1}$ и $\pi_{2}$ обозначают естественные проектирования пространства $X \times Y$ на $X$ и $Y$. Под общей задачей Монжа-Канторовича мы понимаем экстремальную задачу, состоящую в нахождении оптимального значения

$$
\mathscr{C}\left(c ; \sigma_{1}, \sigma_{2}\right):=\inf \left\{c(\mu): \mu \in \Gamma\left(\sigma_{1}, \sigma_{2}\right)\right\},
$$

где $c(\mu)$ - интеграл функции $c$ по $\mu$ (считаем, что по определению $c(\mu)=$ $+\infty$, если $\left.c_{+}(\mu)=+\infty\right)$, а $\Gamma\left(\sigma_{1}, \sigma_{2}\right)$ - множество положительных борелевских мер $\mu$ на $X \times Y$, для которых $\sigma_{1}$ и $\sigma_{2}$ являются их маргинальными мерами: $\pi_{1}(\mu)=\sigma_{1}, \pi_{2}(\mu)=\sigma_{2}$. Положим

$$
\mathscr{V}\left(c ; \sigma_{1}, \sigma_{2}\right):=\inf \left\{\int_{X} c(x, f(x)) \sigma_{1}(d x): f \in \Phi\left(\sigma_{1}, \sigma_{2}\right)\right\},
$$

где $\Phi\left(\sigma_{1}, \sigma_{2}\right)$ — множество сохраняющих меру отображений $f: X \rightarrow Y$. Борелевское отображение $f$ называется сохраняющим меру, если $f\left(\sigma_{1}\right)=\sigma_{2}$,

^ Работа выполнена при поддержке РФФИ, грант 96-01-00336. 
т. е. $\sigma_{1}\left(f^{-1}\left(B_{2}\right)\right)=\sigma_{2} B_{2}$ для каждого борелевского множества $B_{2} \subseteq Y$. Отображение $f \in \Phi\left(\sigma_{1}, \sigma_{2}\right)$ порождает меру $\mu_{f}=\left(\mathrm{id}_{X} \times f\right)\left(\sigma_{1}\right) \in \Gamma\left(\sigma_{1}, \sigma_{2}\right)$ и $c\left(\mu_{f}\right)=\int_{X} c(x, f(x)) \sigma_{1}(d x)$. Следовательно,

$$
\mathscr{C}\left(c ; \sigma_{1}, \sigma_{2}\right) \leqslant \mathscr{V}\left(c ; \sigma_{1}, \sigma_{2}\right) .
$$

Неравенство (3), как правило, оказывается строгим (во многих случаях $\Phi\left(\sigma_{1}, \sigma_{2}\right)$ вообще пусто и, стало быть, $\left.\mathscr{V}\left(c ; \sigma_{1}, \sigma_{2}\right)=+\infty\right)$.

Нас интересуют случаи, когда (3) выполнено со знаком равенства и в задаче (1) существует единственная оптимальная мера, порождаемая отображением $f \in \Phi\left(\sigma_{1}, \sigma_{2}\right)$. Если $X=Y$ - метрический компакт, а $c(x, y)$ его метрика, (1) преврашается в классическую задачу Монжа-Канторовича, являющуюся расширением (релаксацией) старинной задачи Монжа «о выемках и насыпях» [1]. Задача Монжа - это задача (2), в которой $X$ и $Y$ равнообъемные области в $\mathbb{R}^{n}, c$ - евклидово расстояние, а $\sigma_{1}$ и $\sigma_{2}$ - ограничения $n$-мерной меры Лебега на $X$ и $Y$. Существование оптимального отображения в задаче Монжа получено Аппелем в 1887 г. В наше время Судаков [2] получил обобщение теоремы Аппеля для $c(x, y)=\|x-y\|$ (норма не обязательно евклидова) и абсолютно непрерывных $\sigma_{1}$ и $\sigma_{2}$. При этом оптимальная мера неединственна. Недавно Ганжбо и Мак-Канн [3] доказали существование оптимального отображения, эквивалентность задач (1) и (2) и единственность оптимальной меры при условии, что $X=Y=\mathbb{R}^{n}, \sigma_{1}$ абсолютно непрерывна по $n$-мерой мере Лебега и $c(x, y)=h(x-y)$, где $h$ неотрицательная строго выпуклая функция на $\mathbb{R}^{n}$, удовлетворяющая специальным предположениям. Случай неотрицательной квадратической функции $h$ исследовался ранее рядом авторов; соответствующая литература приведена в [3].

В настоящей заметке существование оптимального отображения, эквивалентность задач (1) и (2) и единственность оптимальной меры получены в весьма обшей ситуации и для широкого класса функций стоимости.

2. Основная теорема. Далее $X$ - замыкание открытого множества в $\mathbb{R}^{n}$, а $Y$ - произвольное польское пространство. Нам понадобятся следующие условия:

$\left(A_{1}\right)$ мера $\sigma_{1}$ аб́солютно непрерывна по $n$-мерной мере Лебега;

$\left(A_{c}\right)$ если для $y_{1}, y_{2} \in \operatorname{supp} \sigma_{2}$ функции $c\left(\cdot, y_{1}\right)$ и $c\left(\cdot, y_{2}\right)$ дифференцируемы в некоторой точке $x \in \operatorname{int} X$ и их градиенты в $x$ совпадают, то $y_{1}=y_{2}$.

Условие $\left(A_{c}\right)$ выполнено, в частности, когда $Y=X$ и $c(x, y)=h(x-y)$, где $h$ строго выпукла или строго вогнута.

ТЕОРема 1. Предположим, что величина $\mathscr{C}\left(c ; \sigma_{1}, \sigma_{2}\right)$ конечна и в задаче (1) существует оптимальная мера. Тогда она единственна и порождаетсл единственным (с точностью до $\sigma_{1}$-эквивалентности) отображением $f \in \Phi\left(\sigma_{1}, \sigma_{2}\right)$ в каждом из следуюших случаев: (I) выполнены условия $\left(A_{1}\right)$, $\left(A_{c}\right), X$ выпукло и либо все $c(\cdot, y)\left(y \in \operatorname{supp} \sigma_{2}\right)$ вогнутыл, либо все они выпукльг; (II) выполнено $\left(A_{1}\right), \operatorname{supp} \sigma_{2}$ компактно, int supp $\sigma_{1}$ непусто, причем mes $\left(\operatorname{supp} \sigma_{1} \backslash \operatorname{int} \operatorname{supp} \sigma_{1}\right)=0$ (mes обозначает $n$-мерную меру Лебега), функции $c(\cdot, y)\left(y \in \operatorname{supp} \sigma_{2}\right)$ дифференцируемь на int supp $\sigma_{1}$, причем если $\nabla_{x} c\left(x, y_{1}\right)=\nabla_{x} c\left(x, y_{2}\right)$ в некоторой точке $x \in \operatorname{int} \operatorname{supp} \sigma_{1}$, mо $y_{1}=y_{2}$, 
и все $c(\cdot, y)\left(y \in \operatorname{supp} \sigma_{2}\right)$ локально липшичевы на $\operatorname{supp} \sigma_{1}$ равномерно по $y \in \operatorname{supp} \sigma_{2}$.

Существование оптимальной меры в задаче (1) - это отдельный (и более простой) вопрос. См. [4, Theorem 9].

3. Вспомогательные результаты. Пусть $X$ - произвольное множество и $L-$ некоторое множество вешественных функций на $X$. Многозначное отображение $F: X \rightarrow L$ назовем $L$-циклически монотонным, если для каждого цикла $x_{1}, \ldots, x_{m}, x_{m+1}=x_{1}$ в $\operatorname{dom} F:=\{x: F(x) \neq \varnothing\}$ и любых $l_{k} \in F\left(x_{k}\right)$ выполнено неравенство

$$
\sum_{k=1}^{m}\left(l_{k}\left(x_{k}\right)-l_{k}\left(x_{k+1}\right)\right) \geqslant 0 .
$$

Заменяя в $(4) \geqslant$ на $\leqslant$, получаем определение $L$-циклически антимонотонного отображения. $L$-циклически монотонные отображения - это обобщение классических циклически монотонных отображений [5], а $L$-циклически антимонотонные отображения - обобщение так называемых $c$-циклически антимонотонных отображений [6].

Функция $U: X \rightarrow \mathbb{R} \cup\{+\infty\}$ называется $L$-выпуклой, если она допускает представление $U(x)=\sup \{l(x)-\alpha:(l, \alpha) \in A\}, x \in X$, где $A=A(U)-$ непустое подмножество в $L \times \mathbb{R}$. Множество

$$
\partial_{L} U(x):=\{l \in L: l(z)-l(x) \leqslant U(z)-U(x) \forall z \in X\},
$$

где $U L$-выпукла и $x \in \operatorname{dom} U:=\{z \in X: U(z)<+\infty\}$, называется $L$-субдифференциалом функции $U$ в $x$. Для $x \notin \operatorname{dom} U$ считаем по определению $\partial_{L} U(x)=\varnothing$.

Аналогично определяются $L$-вогнутая функция $V: X \rightarrow \mathbb{R} \cup\{-\infty\}$ и ее $L$-супердифференциал $\partial^{L} V(x)$ в $x \in \operatorname{dom} V:=\{z: V(z)>-\infty\}$. При этом $\partial_{(-L)}(-V)=\partial^{L} V$.

Пусть $F: X \rightarrow L-$ многозначное отображение, $Z:=\operatorname{dom} F$. Рассмотрим на $Z \times Z$ две функции стоимости

$$
\begin{aligned}
& \varphi_{F}\left(z_{1}, z_{2}\right)=\varphi_{F, L}\left(z_{1}, z_{2}\right):=\inf \left\{l\left(z_{1}\right)-l\left(z_{2}\right): l \in F\left(z_{1}\right)\right\}, \\
& \psi_{F}\left(z_{1}, z_{2}\right)=\psi_{F, L}\left(z_{1}, z_{2}\right):=\inf \left\{l\left(z_{1}\right)-l\left(z_{2}\right): l \in F\left(z_{2}\right)\right\}
\end{aligned}
$$

и отметим тождество $\psi_{F, L}\left(z_{1}, z_{2}\right)=\varphi_{-F,-L}\left(z_{2}, z_{1}\right)$.

$\mathrm{C}$ каждой функцией $c: Z \times Z \rightarrow \mathbb{R}$ ассоциируется множество

$$
Q_{0}(c):=\left\{u \in \mathbb{R}^{Z}: u\left(z_{1}\right)-u\left(z_{2}\right) \leqslant c\left(z_{1}, z_{2}\right) \forall z_{1}, z_{2} \in Z\right\} ;
$$

условия непустоты $Q_{0}(c)$ даны в $[7,8]$.

Teоpema 2. Пусть $F: X \rightarrow L, Z=\operatorname{dom} F$.

(I) Следующие утверждения равносильны: (а) $F$ L-ииклически монотонно; (б) $Q_{0}\left(\varphi_{F}\right)$ непусто.

(II) Пусть $F \quad L$-ииклически монотонно. Тогда $u \in Q_{0}\left(\varphi_{F}\right)$, если и только если $u=U \mid Z$, где $U: X \rightarrow \mathbb{R} \cup\{+\infty\} \quad L$-выпукла и $F(z) \subseteq \partial_{L} U(z)$ для всех $z \in Z$. 
ЗАмЕчАния. 1. Отсюда вытекает аналогичная теорема, формулировка которой получается при замене $L$-циклической монотонности, $L$-выпуклости и $\varphi_{F}$ на $L$-циклическую антимонотонность, $L$-вогнутость и $\psi_{F}$.

2. Объединяя (I) и (II), получаем абстрактный вариант известной теоремы Рокафеллера: $F$ является $L$-циклически монотонным тогда и только тогда, когда существует $L$-выпуклая функция $U$, такая, что $F(x) \subseteq \partial_{L} U(x)$ для любой $x \in X$.

Далее $X$ - замыкание открытого множества в $\mathbb{R}^{n}$. Рассмотрим следующие условия на $X$ и $L$ :

$\left(H_{1}\right) \quad X$ выпукло и все $l \in L$ выпукль и полунепрерывны снизу;

$\left(H_{1}^{\prime}\right) \quad X$ выпукло и все $l \in L$ вогнуты и полунепрерывны сверху;

$\left(H_{2}\right)$ все $l \in L$ дифферениируемы на int $X$ и для каждой точки $x \in X$ найдутся окрестность $G_{x}$ и иисло $C=C(x)>0$, такие, ито $\left|l\left(x_{1}\right)-l\left(x_{2}\right)\right| \leqslant$ $C\left\|x_{1}-x_{2}\right\|$ при любих $x_{1}, x_{2} \in G_{x}, l \in L$;

$\left(H_{3}\right)$ если две функции $l_{1}$ и $l_{2}$ из $L$ дифференцируемь в некоторой точке $x \in \operatorname{int} X u \nabla l_{1}(x)=\nabla l_{2}(x)$, mo $l_{1}=l_{2}$.

Обозначим через $S(F)$ множество точек $x \in \operatorname{dom} F$, в которых $F$ однозначно.

Теорема 3. Множество $\operatorname{dom} F \backslash S(F)$ имеет лебегову меру нуль в каждом из следуюших случаев: (I) $F$ L-ииклически монотонно и выполнены условия $\left(H_{1}\right),\left(H_{3}\right)$; (II) $F$ L-циклически антимонотонно и выполнень условия $\left(H_{1}^{\prime}\right),\left(H_{3}\right)$; (III) $F$ L-циклически монотонно или L-ииклически антимонотонно, mes $(\operatorname{dom} F \backslash \operatorname{int} \operatorname{dom} F)=0$ и выполнены условия $\left(H_{2}\right),\left(H_{3}\right)$.

4. О доказательствах. Классический вариант теоремы 2 см. в $[7,8]$. В общем случае доказательство аналогично. В доказательстве теоремы 3 используются теорема 2, теорема Радемахера и дифференцируемость почти всюду выпуклых функций на $\mathbb{R}^{n}$. В доказательстве теоремы 1 используется теорема 3 и тот факт (ср. [3]), что оптимальность $\mu \in \Gamma\left(\sigma_{1}, \sigma_{2}\right)$ влечет за собой $L$-циклическую монотонность отображения $F(x)=\{-c(\cdot, y):(x, y) \in$ $\operatorname{supp} \mu\}$ для $L=\left\{-c(\cdot, y): y \in \pi_{2} \operatorname{supp} \mu\right\}$ и $(-L)$-циклическую антимонотонность отображения $-F$.

\section{ЛитеРАтУРА}

1. Канторович Л. В., Акилов Г. П. Функциональный анализ. Наука, М., 1984. 2. Судаков В. H. Труды МИАН, 141 (1976). 3. Gangbo W., McCann R. J. Acta Math., 177, 113-161 (1996). 4. Levin V. L. In: Functional Analysis, Optimization and Mathematical Economics. Oxford University Press, 1990, pp. 141-176. 5. Rockafellar R. T. Pacif. J. Math., 17, 497-510 (1966). 6. Smith C. S., Knott M. J. Multivariate Anal., 40, 328-334 (1992). 7. Левин В. Л. Матем. сб., 181, № 12, 1694-1709 (1990). 8. Levin V. L. Set-Valued Anal., 4, No. 1, 41-65 (1996).

Центральный экономико-математический институт РАН

Поступило в редакцию 6 марта 1997 г. 